\title{
Inter-professional communication in palliative care: general practitioners and specialists in Switzerland
}

\author{
Brigitte Liebig $^{1 *}$ and Chiara Piccini ${ }^{2}$ \\ Switzerland \\ ${ }^{2}$ Institute for Public Communication, Faculty of Communication Sciences, University of Lugano, Switzerland
}

${ }^{1}$ Institute for Research and Development of Collaborative Processes, School of Applied Psychology, University of Applied Sciences and Arts Northwestern,

\begin{abstract}
This article describes challenges of inter-professional communication between doctors in community-based and specialized palliative care from the perspective of general practitioners. Findings are based on empirical data collected by means of focus groups with 91 general practitioners in Switzerland in 2014 . Qualitative analysis of data has been conducted according to the methodological framework of content-analysis. The article highlights that a lack of institutionalized channels of communication and different professional visions of general practitioners and specialists contribute to fragmented information on patients and increased risk of poor quality of care. The authors discuss measures to improve inter-professional communication in primary and specialized palliative care.
\end{abstract}

\section{Introduction}

The aging of western societies and the prevalence of incurable diseases make end-of-life care a complex field of practice. Multiple professionals have to intervene in order to provide assistance to patients, who often suffer of more than one disease at the same time. In this context, successful inter-professional collaboration is a core premise to ensure high quality patient care $[1,2]$.

However, coordination of services and collaboration in this field has been often evaluated as insufficient [3]. In particular, the limits of information sharing have been pointed out as important barriers to the quality of inter-professional collaboration [4]. Requested are better instruments for knowledge transfer among carers [5] in order to avoid negative consequences of coordination failures, such as unnecessary hospital admissions, problems with symptom control and the incapability to uptake the whole range of patient needs [6,7].

Although general practitioners (GPs) have been often identified as guaranteeing continuity of care across multiple clinical settings [8], cultural and organizational barriers limit inter-professional communication between GPs and other professionals [9]. In particular, the complex interplay between autonomous professional practice and specialists or hospital care has been identified as an often overlooked obstacle [2]. First studies report serious communication gaps between hospital care, specialists (e.g. in oncology) and primary care physicians [10].

Collaboration between generalist providers' and nursing homes and hospital-based specialist palliative care has rarely been systematically assessed [11]. This article aims to examine challenges of inter-professional communication as core element of collaboration between GPs and medical specialists, who take care of terminally ill patients in the hospital or in medical surgeries in Switzerland. From the perspective of GPs we analyze in this paper the information flow between doctors in primary and specialized care, and main challenges of information transfer. Based on the analysis of interview data and on previously gained knowledge about the institutional framework of community-based palliative care in Switzerland [12] the article also depicts possible solutions to the perceived challenges.

\section{Methods}

Knowledge is very limited regarding inter-professional communication of GPs and specialists in palliative care [10]. For getting first insight, a qualitative-inductive approach has been chosen to get an in-depth understanding of associated challenges. Data have been collected by means of 12 focus groups with 91 GPs involved into community-based palliative care in Switzerland in 2014. The focus groups followed semi-structured discussion guidelines which contained questions about premises, challenges, and everyday practices of palliative care work in general practice. No issues touching sensitive patient data were included into the guideline; ethical approval was not required for the study.

Focus groups of GPs have been generated with the support of regional institutes of general practice in the German, French and Italian speaking region of Switzerland (namely in canton Lucerne (LU), Vaud (VD) and Ticino (TI)). 64 male and 25 female GPs with an average work experience of nearly 17 years (range 1-42 years) attended the meetings. The French, Italian and German data have been transcribed verbatim and interpreted in either language; in a second step date were translated into English in order to allow comparative analysis across the language regions. Additionally the participants responded to a brief standardized questionnaire, providing basic demographic and work related data (such as work experience, institutional context).

Correspondence to: Brigitte Liebig, School of Applied Psychology, University of Applied Sciences and Arts Northwestern, Switzerland, Tel: +41 6295723 61; E-mail: brigitte.liebig@fhnw.ch

Key words: inter-professional communication, community-based palliative care, general practitioners, medical specialists

Received: April 04, 2017; Accepted: April 24, 2017; Published: April 27, 2017 
Analysis of data has been conducted according to the procedures of qualitative content analysis [13]. This approach allows an in-depth analysis of inter-professional communication from the perspective of GPs and respective concept building. Based on generative questions and sensitizing concepts, the analysis successively intended to elaborate key categories of explanatory character.

Due to the explorative approach of this study the reach of its results is limited and cannot claim general validity. However, the systematic and comparative approach of the content-analysis allowed improving the quality of interpretation. Further, three workshops were conducted in which a selected number of respondents participated voluntarily. The workshops allowed a member check of the results and to improve the quality of the study.

\section{The meaning of inter-professional communication}

In community-based care the communication between GPs and nurses, other health professionals, nursing homes, hospitals and/or doctors in specialized palliative care constitutes a core element of high quality care for patients $[14,15]$. Communication between caregivers is of particular importance when decisions must be taken at the end of life: decision-making in community-based palliative care often concerns the transition from home to hospital, the prescription and dosage of symptoms management drugs, the interruptions of active treatments, or the implementation of invasive treatments, such as reanimation maneuverings or artificial nutrition [16]. However, decision-making puts high requirements on communication, as well as for information management. They are essential elements in course of the mutual coordination of goals and actions, which can only partly be steered by contractual obligations and formal rules [14].

Our analysis starts from the assumption, that in community-based palliative care GPs are of special importance for ensuring the adequate design and flow of work processes, which includes the appropriate allocation of services between care providers as well as across different institutions and settings of care [17]. Communication practices [18] are based on a long lasting history of interactions between GPs and specialists, which however might not fulfil the demands of palliative care settings. Further, we can assume that communication practices of GPs are framed by the specific context in which care is delivered. As such we can consider aspects of work organization, but also more general the institutional framework in which the activity of palliative care is carried on [19], including training and further education or financial incentives. Consequently, we are interested in identifying, which conditions frame inter-professional communication between GPs and specialists in palliative care and might eventually figure as obstacles or support.

Moreover, we understand communication as informed by professional visions, i.e., by mutual professional ways to codify reality [20]. In fact, professional practices of codification can always be recognized in the discourse of professionals, whenever communication is analyzed in detail [21]. In our corpus of data, we therefore look out for accounts of mutual and different professional visions and for their roles in inter-professional communication.

\section{Results}

Community-based palliative care has been developed and strengthened in Switzerland as part of a national strategy since 2010. According to the EACP (2015) report, palliative care in the community ranges beyond average in Switzerland, compared to other European countries, with respect to the payment of home visits and out of hours. However, integrated health care services are not yet guaranteed when the coordination between primary and specialized palliative care is concerned [12]. According to first studies, appropriate structures and procedures to support palliative care across the boundaries of in- and out-patient care seem to lack considerably [10]. While first networks have been developed for a coordinated management of complexity in the field of health care, these approaches have difficulties to find political/institutional legitimation and financial support.

In this context a fragmentation of information about patients can be identified as a most important challenge of inter-professional communication between GPs and specialized doctors: As the analysis of focus groups with GPs shows it constitutes a limiting factor in decision-making and increases risks of poor quality of care.

\section{Fragmented information}

As the doctors report, care at the end of life includes not only the knowledge about multiple aspects of patient's physical state, but also of the situation at home, the various difficulties experienced by the family, the patient's attitude to tolerate pain, or desires about things to do before dying. However, the focus groups highlight that end-of-life care often requires the involvement of a whole network of professional (and non-professional) actors across palliative care sectors. These different actors have partial views of the patient, which are sometimes difficult to merge, and remain fragmented unless specific communicative practices are implemented. As it is illustrated by a segment of a group discussion, especially good decision-making is characterized by the possibility to merge pieces of information distributed among multiple caregivers:

"I recently received a phone call from intensive care for two patients in a decision-making situation, in relation to what to do, whether to support vital functions or not. They called me and, considering everything I knew, together with all that they knew, we succeeded to work in a good way, following desires of patient and family" (TI_GP_GD3: 778-780¹).

In most cases, the communication between GPs and medical specialists already seems effective enough to share information. Nevertheless, interruptions of the information flow are reported that reveal considerably limits of existing communication practices. As groups of GPs report, this is the case, when for example an emergency happens during a temporary unavailability of the attending physician: In these cases, fragmented information can generate negative consequences. Communication gaps between GPs and specialized doctors result in a breakdown of the information flow, and in an interruption of clinical history: "The patient is taken away (...) and you do not see him anymore” (TI_GP_GD2: 1110-1111).

\section{Lack of institutionalized channels of communication}

As GPs describe fragmented information flows are mostly is caused by the lack of institutionalized channels for inter-professional communication, especially between community-based palliative care and hospitals. Communication between actors of these fields still seems mainly based on individual strategies and informal relations between doctors in specific context, formal structures of communication (see

${ }^{1}$ Every quotation is identified in relation to region (TI/VD/LU), relative number of group discussion (GD1/GD2/GD3/GD4), and line numbers. For instance, the quotation marked as "TI_GPs_GD3:778-780" is an extract of the third group discussion that has been conducted with GPs in Ticino. Transcription conventions: $((\ldots))$ indicates omitted words. More in general, text between double brackets indicates the authors' explication of citation; further, (.) indicates short pause in speech. 
Leavitt 1958) do not exist. The challenges of establishing successful communication practices between community-based and specialized care are enforced by differences in the work organization of hospitals and GPs surgery. From the perspective of GPs, the organization of communication in hospitals does not only follow specific rules, but does not take the restricted availabilities of GPs into account:

GP1: "I don't know (.) if you should be (there) between 4 and $5 \mathrm{pm}$ and you don't go, if they think this shows disinterest over there (.) or if they understand it $((\ldots))$ but it is almost not possible for us to plan such a meeting

GP2: $m h m$ (.) I think they, they say it clearly (.) they cherish it pretty much when you come

\section{GP3: yes, yes}

GP2: but they understand, if you don't come, it is pretty much normal that you don't go, but than if you come (.) they say, this is very, very important for us" (LU_GP_GD2: 958-965)

The fact that GPs are not supposed to participate in hospital case conferences is considered as highly problematic, and due to the selfunderstanding of specialized palliative care, which contacts GPs only when all treatment possibilities have been exhausted and the patient is terminally ill. But the possibility of GPs for communication with hospital doctors already is even more basically limited, because of remuneration system constraints for Swiss doctors working in a local doctors' surgery [12]: GPs need to invoice each individual medical service choosing among a list which does not include time for participating in family conferences and meetings with other professionals. However, as GPs report, obstacles for joining multidisciplinary meetings are primarily related to work organization, and not due to remuneration, because it often interferes with their duties in GP surgery.

\section{Different professional visions}

GPs perceive challenges not only in the communication with hospital doctors, but also in communicating with specialists who treat outpatients, in particular oncology patients. Also in this case, information flow is interrupted, since GPs do not have access to clinical nor psycho-social information about patients during the time in which they are mainly followed by specialized doctors. Further challenges seem related to different professional visions: GPs stress the difference between their own professional philosophy - which is often oriented toward a "holistic care" of patients - and the perspective of medical specialists, which GPs perceive as rather focused on "segments of the body" and on "winning" against specified pathologies. The following part of a group discussion illustrates the challenges of finding an inter-professional agreement about appropriate treatment between specialists and GPs.

"This really is a difficult tightrope walk (.) how far should one intervene (.) in favor of the patient, the patient trusts in both (.) in the GP as well as the specialist and (.) it is incredibly difficult to say something critically about a colleague or when you just don't agree with it, then you just have to watch out, or it comes back to you like a boomerang. (.) So normally, I'm very cautious and accept it ((laughs)) unless he is wide of the mark but (.) at the end the patients have to decide by themselves how far they want to go with the pain, so how far they want to get therapy (LU_GP_GD1: 980-998)

As this doctor describes, medical advices by GPs seem quite often experienced as critique that interfere with the decision making process, instead of a resource. Comments of GPs rather seem to cause conflicts - if not becoming a "boomerang", which could hit back. Since a discussion between GPs and specialists does not seem possible, and since synergy of vision cannot be achieved, the patient seems finally left to himself/ herself, when it comes to decision-making about medication. The quote also highlights the role of the patient, who does not facilitate the collaboration between the medical professions, when sometimes switching between the considerations and visions of specialists and GPs.

\section{GPs strategies to face challenges}

The institutionalization of communication channels for sharing information between hospital-based and community-based palliative care is described as highly desirable by GPs, but it is also described as rare:

"The collaboration with oncologists is better with ((hospital name)) because oncologists don't hesitate to ask the GP for the blood test. Then oncologists summarize with their group of specialists. The patients like that, because they are going to their GP to whom they are related" (VD_ GP_GD1:260-263).

Due to the lack of formalized communication GPs develop various strategies. In the following quote a doctor describes, how he tries to assure his availability for the patient during his hospital stay: "I usually tell the patient: 'I see a mass on the lung', or: 'we have a head $x$-ray'. And then, I say: 'you will enter in a system, (...) you must know that I'm staying there if you need me"' (VD_GP_GD2:163-167). Also, GPs report trying to overcome pro-actively information breakdowns between themselves and specialists: "I call him ((the oncologist)) at the phone to ask him about the patient or a relative comes to me and asks me to get information" (TI_GP_GD2: 1138-1140).

As focus group discussion show, the initiatives of GPs can result in the development of a collaboration practice, in which both, the specialist and the GP maintain their role toward the patient, offering different and valuable contributions of their professional visions. In particular, when patients are able to discuss the course of action, as it has been proposed by the medical specialist, also with their GP, decisions are possible, which take into account both, the specialist indication and patients personal needs and perspectives:

"A decision is made together with the patient and often that decision is not the one of the specialist, but it is a decision derived from ((the perspective of the specialist)), not in contrast to the specialist but different $((. .)$.$) taking into account the whole clinical and family context, and$ what the patient desires." (TI_GP_GD2: 1092-1100).

\section{Increased risk of poor quality of care}

In our sample GPs describe breakdowns in information flow as a dangerous situation that challenges the quality of care and decisionmaking. This is the case, when a lack of professional agreement results in repetitive treatments that seem unreasonable with reference to the patients' general condition, or when patient's desires remain unsatisfied. The following quote illustrates this case:

"It happens sometimes when we are on holidays that inappropriate courses of actions are taken, as for example to place a defibrillator ((...)) or to change the pacemakers when the patient has a terminal cancer of the thyroid $((\ldots))$. In my opinion, this protocol according to which you have to change the pacemaker without considering to whom you are changing it, these are serious things" (TI_GP_GD2, 1175-1198). 
Further, negative consequence of information breakdowns between GPs and specialists result - from the perspective of GPs - in a lack of support for relatives quite generally, as well as particularly in decision making.

\section{Discussion}

Based on the analysis of focus groups with GPs from different regions in Switzerland we identified the fragmentation of information across community-based and specialized palliative care as a crucial problem of inter-professional communication and decision-making: As far as we can estimate from our data, communication practices of GPs and specialists are unable to bridge this gap until today. As we assumed, different attitudes and visions of doctors in primary and specialized palliative care as well as the specific conditions of work within these fields contribute strongly to this situation.

In Swiss community-based palliative care, the prevalence of informal strategies based on mutual relationships often allows to surmount the lack of institutional channels for inter-professional communication. However, rare incidents caused by information breakdowns do happen, which suddenly demonstrate the limits of the current organization of work respectively informal communication structures. Such incidents compromise the quality of care and exacerbate sufferings and fatigue - of the patient first.

As GPs stress in our study, any efforts to increase information sharing seem highly desirable to improve the quality of care at the end of life. Quite basically the development of strategies and instruments for knowledge transfer between GPs and other caregivers, namely relatives, home care and hospital nurses, specialized doctors who work in the community, and hospital doctors seems highly welcome. To begin with, inter-professional collaboration and coordination between adjacent areas of practice in community-based and specialized palliative care have to be assessed and optimized; characteristics of work organization (as for example the scheduling of hospital case conferences, and the scheduling in GPs surgery) have to be improved in order to increase interaction opportunities between hospital doctors, specialists and GPs. Informal strategies have to be complemented by formalized channels and tools, which allow for better coordination of outpatient and inpatient care.

However, with respect to previous empirical contributions [5] and communities of practice theory $[22,23]$ which stress the need of continued interactions to develop functional collaboration practices and a sense of mutual understanding, we assume that it will not be enough to improve the institutional framework for communication. Besides communication tools, or the sensitizing of doctors for the crucial meaning of inter-professional communication, professional concepts in primary and specialized care and the self-understanding of actors have to be reflected [24]. Based on this reflection continuous interaction will help professionals to develop collaborative practices, which again can facilitate the alignment between different professional perspectives of doctors involved in end-of-life care.

More basically, we still need more information and knowledge on how possible modes for integrated palliative care can be developed, and which staff, education and further resources are necessary for their development in Switzerland and abroad. Further research is needed, in order to contribute to a deeper understanding of communication and collaboration in palliative care as a highly complex work environment.

\section{Acknowledgments}

This article is based on the study "Decision-making in general practice settings at the end of life ", which has been carried out in the Swiss national research program 67 "end of life" and financed by the Swiss National Research Foundation (project-no.: 406740-139270). We are very grateful for the support of Vanessa Alvarado and Karine Darbellay for their collaboration in research as well as to Klaus Bally, Heike Gudat, Peter Voll and Antonella Carassa for their advices in the course of our work.

\section{References}

1. Gillan PC, Arora S, Sanderson H, Turner L (2013) Palliative care simulation: Nurturing interprofessional collegiality. Health and Interprofessional Practice 2: eP1051.

2. Hartrick Doane G, Stajduhar K, Causton E, Bidgood D, Cox A (2012) End-of-life care and interprofessional communication: Not simply a matter of "more". Health and Interprofessional Practice 1: eP1028.

3. Wittenberg-Lyles EM, Oliver DP, Demiris G, Regehr K (2009) Exploring interpersonal communication in hospice interdisciplinary team meetings. J Gerontol Nurs 35: 38-45. [Crossref]

4. Kiely F, Murphy M, O'Brien T (2012) Information transfer to out-of-hours cooperatives: a survey of general practitioners' views in relation to palliative patients. BMJ Support Palliat Care 3: 405-11. [Crossref]

5. Pringle A, Finucane A, Oxenham D (2014) Improving electronic information sharing for palliative care patients. BMJ Qual Improv Rep 3. [Crossref]

6. Bally K, Lingenhel S., Tschudi P. (2012) High-quality hospital discharge summaries general practitioners expectations. Ther Umsch 69: 5-7. [Crossref]

7. Alsop A (2010) Collaborative working in end-of-life care: developing a guide for health and social care professionals. Int J Palliat Nurs 16:120-125. [Crossref]

8. Oishi A, Murtagh FE (2014) The challenges of uncertainty and interprofessiona collaboration in palliative care for non-cancer patients in the community: A systematic review of views from patients, carers and health-care professionals. Palliat Med 28: 1081-1098. [Crossref]

9. Mitchell GK, Reymond EJ, McGrath BP (2004) Palliative care: promoting general practice participation. Med J Aust 180: 207-208. [CrossRef]

10. Otte I, Jung C, Bally K, Elger B, Schildmann J (2016) Interprofessional Silence at the End of Life: Do Swiss General Practitioners and Hospital Physicians Sufficiently Share Information About Their Patients? J Palliat Med 19: 983-6. [Crossref]

11. Firn J, Preston N, Walshe C (2016) What are the views of hospital-based generalis palliative care professionals on what facilitates or hinders collaboration with in-patien specialist palliative care teams? A systematically constructed narrative synthesis. Palliat Med 30: 240-256. [Crossref]

12. Alvarado V, Liebig B (2015) Conditions of Palliative Home Care: The Case of Family Physicians in Switzerland. Primary Healthcare: Open Access 5: 180.

13. Mayring P (2014) Qualitative content analysis: theoretical foundation, basic procedures and software solution. Klagenfurt. URN: http://nbn-resolving.de/urn:nbn:de:0168ssoar-395173.

14. D’Amour D, Ferrada-Videla M, San Martin Rodriguez L, Beaulieu MD (2005) The conceptual basis for interprofessional collaboration: Core concepts and theoretical frameworks. J Interprof Care 19: 116-131. [Crossref]

15. Gilbert M, Staley C, Lydall-Smith S, Castle D (2008) Use of collaboration to improve outcomes in chronic disease. Disease Management \& Health Outcomes 16: 381-390.

16. van der Heide A, Deliens L, Faisst K, Nilstun T, Norup M, et al. (2003) End-of-life decision-making in six European countries: descriptive study. Lancet 362: 345-350. [Crossref]

17. Brogaard T, Jensen AB, Sokolowski I, Olesen F, Neergaard MA (2011) Who is the key worker in palliative home care? Scand J Prim Health Care 29: 150-156. [CrossRef]

18. Lave J, Wenger E (1991) Situated Learning: Legitimate Peripheral Participation. Cambridge University Press, Cambridge

19. Piccini C, Carassa A, Colombetti M (2006) Narrative activity within an institutional framework: how a team constructs problems that can be solved. Studies in Communication Sciences 6: 327-336. 
Liebig B (2017) Inter-professional communication in palliative care: general practitioners and specialists in Switzerland

20. Goodwin C (1994) Professional vision. American Anthropologist 96: 606-633.

21. Piccini C, Carassa A (2010) Collective decision making in rehabilitation teams: practices of talking work. Bulletin suisse de linguistique appliquée 2: 147-163.

22. EAPC (European Association of Palliative Care) (2015) EAPC White Paper on outcome measurement in palliative care: Improving practice, attaining outcomes and delivering quality services - Recommendations from the EAPC Task Force on Outcome Measurement [on-line] http://www.eapcnet.eu/Portals/0/Clinical/Publications/
PM2015_Bausewein.pdf [10.10.2016].

23. Wenger E (2010) Communities of practice and social learning systems: the career of a concept. In: Blackmore C (Ed.): Social learning systems and communities of practice. London: Springer and Open University, 179-198.

24. Alvarado V, Liebig B (2016) Inter-Professional Collaboration between Family Doctors and Nurses at the End of Life; Challenges of Community-Based Palliative Care in Switzerland. Journal of Community \& Public Health Nursing 2: 124.

Copyright: $(02017$ Liebig B. This is an open-access article distributed under the terms of the Creative Commons Attribution License, which permits unrestricted use, distribution, and reproduction in any medium, provided the original author and source are credited. 